\title{
Monitoreo microbiológico para la gestión ambiental de Aguas Residuales Hospitalarias.
}

\section{Microbiological monitoring for the environmental management of Hospital Wastewater.}

Andrés Beltrán Dávalos 1, Mayra Escudero Vilema. ${ }^{2}$, Silvana Córdova Morales. ${ }^{3}$ \& Carlos Rolando Rosero Erazo. ${ }^{4}$

\begin{abstract}
.
DOI: https://doi.org/10.33262/cienciadigital.v3i3.4..882

The microbiological study to evaluate pathogens in wastewater from the General Puyo Hospital unit was developed in the biochemical and bacteriological analysis laboratories of the Faculty of Sciences of the Polytechnic School of Chimborazo. During three months, 6 composite samples were collected with their respective repetitions before and after the Wastewater Treatment Plant (WWTP), performing physical-chemical analysis such as $\mathrm{pH}$, Conductivity, salinity, total dissolved solids, turbidity, color, $\mathrm{BOD}_{5}, \mathrm{COD}$, followed by a microbiological analysis by means of sowing plaques for the identification and quantification of bacteria: Escherichia Coli, Staphylococcus aureus and Salmonellas spp., and finally the Gram stain was performed before carrying out the biochemical tests where a total of Gram bacilli were identified negative, the bacteria identified were from the family
\end{abstract}

\footnotetext{
1 Escuela Superior Politécnica de andresbeltrandavalos@gmail.com

2 Escuela Superior Politécnica de coprinus2@gmail.com

3 Escuela Superior Politécnica de saplitos1733@hotmail.com

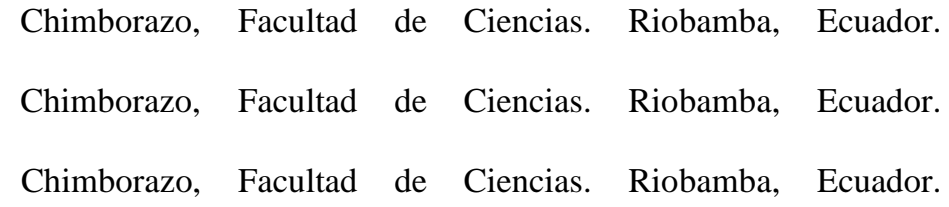


Enterobacteriaceae. Identifying the following families, Escherichia coli, (50\%), Klebsiella pneumoniae, (10\%) Enterobacter cloacae (0.1\%) Shigella dysenteriae, (10\%) Salmonella spp (30\%), thus concluding that the wastewater from This hospital unit has a high percentage of toxicity and conventional wastewater treatments do not eliminate its biological toxicity.

Keywords: Microbiology, Escherichia Coli, Staphylococcus aureus, Salmonellas spp, biochemical tests.

\section{Resumen.}

El estudio microbiológico para evaluar patógenos en aguas residuales de la unidad Hospitalaria General Puyo se desarrolló en los laboratorios de análisis bioquímicos y bacteriológicos de la Facultad de Ciencias de la Escuela Superior Politécnica de Chimborazo. Durante tres meses se recolectó 6 muestras compuestas con sus respectivas réplicas antes y después de la Planta de tratamiento de Aguas Residuales (PTAR) realizando análisis físico - químico como: $\mathrm{pH}$, Conductividad, salinidad, sólidos disueltos totales, turbiedad, color, DBO5, DQO, seguido de un análisis microbiológico mediante siembras de vertido en placa para la identificación y cuantificación de bacterias: Escherichia Coli, Staphylococcus aureus y Salmonellas spp .y finalmente se realizó la Tinción Gram previo a realizar las pruebas bioquímicas donde se identificaron una totalidad de Bacilos Gram negativos, las bacterias identificadas fueron de la familia Enterobacteriaceae. Identificando las siguientes familias, Escherichia coli, (50\%), Klebsiella pneumoniae, (10\%) Enterobacter cloacae $(0,1 \%)$ Shigella dysenteriae, (10\%) Salmonella spp (30\%), concluyendo así que las aguas residuales provenientes de esta unidad Hospitalaria tienen un porcentaje de toxicidad alta y los tratamientos convencionales de aguas residuales no eliminan la su toxicidad biológica..

Palabras claves: Microbiología, Escherichia Coli, Staphylococcus aureus, Salmonellas spp, pruebas bioquímicas.

\section{Introducción.}

Las aguas residuales hospitalarias se consideran como una de las principales fuentes de contaminantes emergentes como resultado de las actividades que se realizan en los establecimientos de salud (Grisales, Ortega, \& Rodriguez, 2011). El agua residual de un establecimiento hospitalario es una mezcla compleja capaz de generar serios problemas 
ambientales, pudiendo llegar a ser de cinco a quince veces más toxica que las aguas residuales domésticas (Magdaleno et al., 2012; Ramos Alvariño, 2009).

Los hospitales son considerados como la mayor fuente de contaminantes emergentes como residuos de laboratorio, excreción de los pacientes, actividades de investigación, entre otros (Gil, Soto, Usma, \& Gutiérrez, 2012; Ramírez-Sánchez, Martínez-Austria, Quiroz-Alfaro, \& Bandala, 2015). Uno de los principales problemas causados por los efluentes hospitalarios se debe a su descarga en los sistemas de alcantarillado urbano (Marín et al., 2009).

Los riesgos que pueden generar los líquidos hospitalarios que se vierte en el sistema de alcantarillado sin previo tratamiento son: posibles efectos patogénicos de numerosos microorganismos y los efectos tóxicos y genotóxicos de una gran variedad de sustancias químicas generadas en los hospitales (Balcioglu, Zhang, \& Tarazi, 2003).

Los efluentes que se producen en unidades médica se identifica las siguientes tipologías de patógenos: la Klebsiella spp. causa infección del tracto urinario en pacientes hospitalizados en América Latina, lo que demostró una alta prevalencia de resistencia en infecciones de alta frecuencia (Tejada Llacsa et al., 2015), de forma similar, el análisis de los Enterobacteriaceae en hospitales de Brasil entre 1997 y 2003 demostraron que el 50\% de las Klebsiella pneumoniae y el 18\% de las E. coli eran positivas para el betalactamasa de espectro extendido (BLEE) (Claudio, Nathanael D, \& Mark P, 2015).

La Shigella dysenteriae es un bacilo Gram negativo perteneciente a la familia Enterobacteriácea que se encuentra estrechamente relacionada con el género Escherichia. S. dysenteriae que afecta el intestino y el sistema nervioso central, suele ocasionar cuadros y complicaciones graves, que incluyen megacolon tóxico y síndrome urémico-hemolítico; las tasas de letalidad han llegado a 20\% entre los casos hospitalizados (León-Ramírez, 2002; Pupo, Lan, \& Reeves, 2002).

Las bacterias patógenas Staphylococcus aureus por su capacidad para crecer en presencia de $6,5 \%$ de cloruro de sodio, a $\mathrm{pH} 9,6$, entre 10 y $45^{\circ} \mathrm{C}$ son bacterias Gram negativas, fisiológicamente relacionadas con las bacterias lácticas que dan negativa la prueba de la catalasa, se afirma que el crecimiento de este patógeno es alta en temperaturas relativamente bajas, aunque existe crecimiento en menor proporción en temperaturas altas. 
Los Staphylococcus aureus, produce lesiones superficiales de la piel y abscesos localizados en otros sitios, causa infecciones del sistema nervioso central e infecciones profundas como osteomielitis y endocarditis (Leung, 2014), es causante de infecciones respiratorias como neumonía, infecciones del tracto urinario, y es la principal causa de infecciones nosocomiales.(García Apac, 2011; Leung, 2014).

El patógeno Escherichia coli mantiene una mayor resistencia a ampicilina con un 34.0\% de bacterias coliformes resistentes, seguida por las bacterias resistentes a cefalotina (17.0\%) y alcanzando valores máximos de $100.0 \%$ y $37.0 \%$ respectivamente. Con respecto a los bacilos Gram negativos no coliformes, también se detectó una mayor resistencia a ampicilina, seguida por la cefalotina alcanzando valores máximos de $87.0 \%$ y $40.0 \%$ respectivamente. Los aislamientos de coliformes resistentes fueron identificados los que presentaron multiresistencia (Mosquito, Ruiz, Bauer, \& Ochoa, 2011).

La Salmonella spp. pertenece a la familia Enterobacteriácea, son bacilos Gram negativos causantes de salmonelosis, una gastroenteritis autolimitada, lo que conduce a enfermedades invasivas que requieren tratamiento antimicrobiano (Terrier \& Martinez, 2012).

Generalmente las plantas de tratamiento emplean procesos biológicos sin mostrar resultados sobre aguas de tipo residual hospitalaria, ya que su capacidad de remoción en este tipo de compuestos recalcitrantes es baja. En general la presencia de residuos farmacéuticos en el ambiente y en los sistemas acuáticos, constituyen un serio problema ya que son extremadamente resistentes a la degradación biológica y usualmente escapan intactos al tratamiento de plantas convencionales (Javier Benitez, Acero, Real, \& Roldán, 2009).

Por los antecedentes mencionados resulta transcendental plantear una investigación que permita evaluar en qué medida se encuentra contaminando estos residuos líquidos hospitalarios que son vertidos al acueducto que conduce hacia la planta de Tratamiento y determinar en qué grado de incumplimiento en que se encuentran tales residuos.

Las aguas residuales presentan gran cantidad de carga microbiana que puede causar problemas a la salud de las personas que al ponerse en contacto con los compuestos y sustancias de otras áreas de los hospitales pueden poseer la capacidad de producir mutaciones en los patógenos presentes en el agua generando efectos genotóxicos por lo que su 
determinación mediante pruebas bioquímicas es fundamental para la selección del tipo de tratamiento.

\section{Metodología}

El Hospital General Puyo, se encuentra ubicado en el perímetro 9 de Octubre y Bolívar Feicán (Provincia Pastaza). Situado en la zona central de la Región Amazónica a 7640’ y $78^{\circ} 10^{\prime}$ de longitud oeste y entre los $1^{\circ} 10^{\prime}$ y $2^{\circ} 35^{\prime}$ de latitud sur, donde se realizó tres muestreos compuestos de aguas residuales con tres réplicas cada uno antes del tratamiento convencional de la PTAR y después de su descarga al sistema de alcantarillado.

Un volumen de $250 \mathrm{~mL}$ para análisis microbiológicos fueron recolectados y $1000 \mathrm{~mL}$ para análisis complementarios de DBO5, DQO, pH, temperatura y Salinidad. El muestreo, manejo y conservación de muestras de las aguas residuales, se realizó bajo los criterios técnicos establecidos en la Norma Técnica Ecuatoriana NTE INEN 2169:2013 y el Manual de métodos analíticos para la determinación de parámetros fisicoquímicos básicos en aguas (APHA/AWWA/WEF, 2012).

\section{Técnica para crecimiento bacteriano (Escherichia Coli, Salmonella spp, Staphylococcus} aureus).

La preparación de cultivos considera criterios de: esterilización, solvente (agua desionizada) y proporciones sugeridas por el fabricante de los cultivos (Bécares, 2014). Los medios de cultivo utilizados para el crecimiento de las bacterias: Escherichia Coli, Salmonella spp, Staphylococcus aureus son: Brilliant Green Bile y BBL Eosin, Agar SS, (BBL)TM Mannitol Salt Agar.

Una vez diluidos en agua desionizada se los llevo aun reverbero hasta que alcance por tres veces el punto de ebullición esto para garantizar una dilución total, posteriormente se colocan los medios en el autoclave para su esterilización, una vez esterilizados se procede a retirar los medios y colocarlos en tubos de ensayo en el caso del agar Brilliant Green Bile colocando $9 \mathrm{~mL}$ por tubo, mientras que los otros medios se colocan en placas Petri en una cantidad de $20 \mathrm{~mL}$ y se los deja reposar hasta que adquieran una óptima temperatura y se solidifiquen. Una vez listas las cajas Petri con los medios se procede a colocar un mililitro de muestra diluida previamente en agua de peptona (agua residual-hospital). Incubamos en una estufa a 
$37^{\circ} \mathrm{C}$ durante un periodo de tiempo de 24 a 48 horas y procedemos al conteo (Mackenzie, Taylor, \& Gilbert, 2009)

\section{Técnicas para realizar las pruebas bioquímicas}

La Tinción Gram fue el primer método utilizado en esta investigación, tomando un pequeño inoculo de la colonia bacteriana deseada y se realiza un frotis amplio sobre la placa porta objetos, esperando unos segundos y flameando rápidamente 3 veces para fijar la muestra.

Se colocó en la placa, cristal violeta por 2 minutos se lavó con agua destilada, se coloca una solución de Lugol por 1 minuto y su respectivo lavado, adicionamos alcohol acetona al 30\% por 30 segundos se lava y después se colocó safranina por un minuto se lavó la placa y dejo secar al aire.

Para la identificación de pruebas bioquímicas se utilizaron medios de cultivo como: agar Kligler el cual se preparó 2.08gr en $40 \mathrm{ml}$ de agua destilada, agar Simmons Citrato $0.968 \mathrm{~g}$ en $40 \mathrm{~mL}$ de agua destilada, agar base Urea, se preparó $0.84 \mathrm{~g}$ en $40 \mathrm{~mL}$ de agua destilada, agar SIM (Sulfuro - Indol - Movilidad) $1.12 \mathrm{~g}$ se prepararon en $40 \mathrm{~mL}$ de agua destilada (Pablo García Blancas, 2014).

Una vez diluidos los medios en agua destilada y esterilizados se procedió a la siembra colocando en tubos de ensayo la cantidad de $5 \mathrm{~mL}$ utilizando la técnica pico de flauta dejando que se solidifiquen los tres primeros medios y el ultimo deberá solidificarse en forma vertical. Para la siembra se procede a introducir una asa de platino en la muestra (agua residualhospital) empleando la técnica de siembra en estría en la superficie inclinada, en los tubos verticales se realizó la siembra por punción profunda. Se selló y etiquetó e incubó a una temperatura óptima de $37^{\circ} \mathrm{C}$ por un periodo de 24- 48 horas (FONTALVO, 2018; Pablo García Blancas1, 2014).

\section{Resultados}

Se identificó a nivel específico las Colonias Escherichia coli., Colonias Salmonella spp. y Colonias Staphylococcus aureus. no presentando diferencias entre las reportadas a la entrada de la planta y salida como lo demuestra la tabla 1, su descripción e interrelación con 
parámetros físicos, físico - químicos y químicos se la detalla en función del patógeno determinado.

Tabla 1: Resultado de Colonias microbianas de aguas residuales del Hospital Puyo.

\begin{tabular}{lllllll}
\hline \multicolumn{5}{c}{ RESULTADOS PROMEDIOS DE ANALISIS MICROBIOLOGICO } \\
\hline \multirow{3}{*}{ E.P. T } & C. E.c & UFC & C.S. spp. & UFC & C. S. a. & UFC \\
\cline { 2 - 7 } & 100 & $>1 \times 10^{6}$ & 79 & $7,9 \times 10^{5}$ & 45 & $4,5 \times 10^{5}$ \\
\hline \multirow{2}{*}{ S.P. T } & 100 & $>1 \times 10^{6}$ & 81 & $8,1 \times 10^{5}$ & 49 & $4,9 \times 10^{5}$ \\
\cline { 2 - 6 } & 100 & $>1 \times 10^{6}$ & 82 & $8,2 \times 10^{5}$ & 46 & $4,6 \times 10^{5}$ \\
\hline & 100 & $>1 \times 10^{6}$ & 84 & $8,4 \times 10^{5}$ & 49 & $4,9 \times 10^{5}$ \\
\hline
\end{tabular}

E.P.T: Entrada planta de tratamiento. S.P.T: Salida planta de tratamiento. C.E.c: Colonias Escherichia coli. C.S.ssp: Colonias Salmonella spp. C.S.a: Colonias Staphylococcus aureus.

\section{Escherichia Coli.}

El DBO5 en un rango de (102 - 110) mg/L presentan un crecimiento de $(100,000)$ UFC, tanto de la entrada como salida de la planta de tratamiento de dicho hospital. El DQO dentro del rango de $(200$ - 280) $\mathrm{mg} / \mathrm{L}$ influyen en el crecimiento a la entrada de la planta de tratamiento presentando un crecimiento de cepas bacterianas de $(100,000)$ UFC.

En la salida de la planta de tratamiento existe una mayor frecuencia de crecimiento bacteriano dentro de los mismos rangos equivalentes a $(100,000)$ UFC.

\section{Salmonella spp.}

En ell parámetro DBO5 hay crecimiento en el siguiente rango $(<102-110) \mathrm{mg} / \mathrm{L}$ cepas entre $(<50,000-100,000)$ UFC, los rangos de crecimiento en cuanto a DQO de $(200-280) \mathrm{mg} / \mathrm{L}$ mantiene un crecimiento (> 50,000 - > 250,000) UFC, para la entrada de la planta de tratamiento en menor frecuencia, mientras en la salida de la planta presenta una mayor frecuencia de crecimiento equivalente a (> 50,000 - > 250,000).

\section{Staphylococcus aureus.}

El rango de DQO5 (> 102 - 110) mg/L en la entrada de la planta de tratamiento presenta un crecimiento de cepas bacterianas $(20,000$ - 50,000) UFC, mientras que en la salida de la planta de tratamientos el crecimiento de cepas bacterianas es de $(>40,000-100,000)$ UFC. 
Otro parámetro medido en aguas residuales del Hospitales General Puyo es el DBO mostrando los siguientes datos en rangos de crecimiento (200- 280) $\mathrm{mg} / \mathrm{L}$ presentando en la entrada de la planta de tratamientos la mayor frecuencia de crecimiento en el rango de DQO (220-235) $\mathrm{mg} / \mathrm{L}$, las cepas bacterianas crecieron en un rango de (20,000-40,000) UFC, a diferencia de la salida de la planta de tratamiento que presenta un crecimiento de $(20,000$ 100,000) UFC la mayor frecuencia de crecimiento se presentó en el rango de DQO (225-235) $\mathrm{mg} / \mathrm{L}$.

\section{Identificación de patógenos asociados a las aguas residuales del hospital}

Las pruebas bioquímicas de identificación indicadas en la tabla 2, fueron las siguientes. Según Álvarez, la prueba de Kliger comprueba la capacidad de una bacteria para metabolizar glucosa y lactosa; la prueba SIM la capacidad de degradar triptófano a Indol; la prueba de citrato, la capacidad de utilizar esta sustancia como única fuente de carbono y por último la prueba de Urea, la capacidad de desdoblar esta sustancia por un proceso de alcalinización (Gil et al., 2012; Grisales et al., 2011).

Tabla 2: Resultados Pruebas bioquímicas.

\begin{tabular}{|c|c|c|c|c|c|c|c|c|c|c|c|}
\hline \multirow[t]{2}{*}{ Color } & \multicolumn{4}{|c|}{ Agar Kliger } & \multicolumn{3}{|c|}{ Agar SIM } & \multirow{2}{*}{ 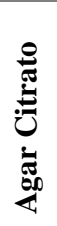 } & \multirow{2}{*}{ 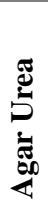 } & \multirow{2}{*}{$\begin{array}{c}\text { Tipo de } \\
\text { Microorganismos }\end{array}$} & \multirow{2}{*}{$\begin{array}{c}\% \\
\text { crecimiento }\end{array}$} \\
\hline & $\stackrel{\mathscr{I}}{\mathbf{I}}$ & 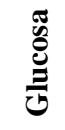 & 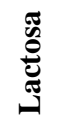 & שี & $\stackrel{\mathscr{I}}{\mathbb{I}}$ & $\vec{e}$ & $\overrightarrow{\vec{e}}$ & & & & \\
\hline Amarillo + & - & + & + & + & + & - & - & - & - & Escherichia coli & $50 \%$ \\
\hline Amarillo + & - & + & + & + & + & - & - & + & + & $\begin{array}{l}\text { Klebsiella } \\
\text { pneumoniae }\end{array}$ & $10 \%$ \\
\hline Amarillo + & - & + & + & + & - & + & - & + & - & Enterobacter cloacae & $0,1 \%$ \\
\hline $\begin{array}{l}\text { Pico rojo + fondo } \\
\text { amarrillo }\end{array}$ & - & + & - & + & + & - & - & - & - & Shigella dysenteriae & $10 \%$ \\
\hline Negro & + & + & - & + & - & + & - & + & - & Salmonella spp. & $30 \%$ \\
\hline
\end{tabular}

$\mathrm{H}_{2} \mathrm{~S}$ : ácido sulfhídrico. Mov: Movilidad. Mot: Motilidad.

La caracterización de las bacterias se determinó mediante la diferenciación de géneros Enterobacteriaceae. Las especies encontradas fueron las siguientes familias, Escherichia coli, 
(50\%), Klebsiella pneumoniae, (10\%) Enterobacter cloacae $(0,1 \%)$ Shigella dysenteriae, (10\%) Salmonella spp (30\%).

\section{Conclusión}

- Es evidente que en las condiciones actuales de la planta de tratamiento de aguas residuales del Hospital Puyo presenta una contaminación excesiva de patógenos en aguas determinando que el tratamiento brindando a estos efluentes no es adecuado pues prevalece la presencia del patógeno Escherichia coli que arroja un valor de crecimiento bacteriano de 4,9x 105 UFC, de Salmonella spp, presenta un crecimiento de 8,4x 105 UFC y de Staphylococcus aureus de 4,9x 105 UFC contrastándose con el crecimiento relativo de bacterias patógenas causantes de una toxicidad extremadamente alta en los seres humanos como: Escherichia coli, (50\%), Klebsiella pneumoniae, (10\%) Enterobacter cloacae $(0,1 \%)$ Shigella dysenteriae, (10\%) Salmonella spp. (30\%) evidencias que aseguran el grado de contaminación y la resistencia que presenta estas bacterias patógenas a tratamientos convencionales aplicados a estos efluentes generados por la unidad de salud.

\section{References Bibliogràficas}

APHA/AWWA/WEF. (2012). Standard Methods for the Examination of Water and Wastewater. Part 1000. Standard Methods. https://doi.org/ISBN 9780875532356

Balcioglu, A., Zhang, K., \& Tarazi, F. I. (2003). Dopamine depletion abolishes apomorphineand amphetamine-induced increases in extracellular serotonin levels in the striatum of conscious rats: A microdialysis study. Neuroscience. https://doi.org/10.1016/S03064522(03)00219-7

Bécares, E. (2014). La Biotecnología Ambiental, ¿la cenicienta de la Biotecnología? Ambiociencias-Revista de Divulgacion

Cientifica. https://doi.org/10.3989/arbor.2014.768n4011

Claudio, R., Nathanael D, R., \& Mark P, S. (2015). Resistencia emergente a los antibióticos: una amenaza global y un problema crítico en el cuidado de la salud. Rev Peru Med Exp Salud Publica, 32(1), 139-145. https://doi.org/10.17843/rpmesp.2015.321.1586 
FONTALVO, J. L. (2018). PRUEBAS BIOQUÍMICAS PARA LA IDENTIFICACIÓN DE

BACTERIAS. In Manual de practicas de laboratorio de Microbiología. https://doi.org/10.2307/j.ctt1zk0mfb.8

García Apac, C. (2011). Staphylococcus aureus meticilino resistente adquirido en la comunidad. Acta Médica Peruana.

Gil, M. J., Soto, A. M., Usma, J. I., \& Gutiérrez, O. D. (2012). Contaminantes emergentes en aguas, efectos y posibles tratamientos. Emerging Contaminants in Waters: Effects and Possible Treatments.

Grisales, P., Ortega, J., \& Rodriguez, T. (2011). Aguas Residuales Hospitalarias Aplicando Ozono Removal of Organic Matter and Toxicity in Hospital Wastewaters By Ozone. Dyna.

Javier Benitez, F., Acero, J. L., Real, F. J., \& Roldán, G. (2009). Ozonation of pharmaceutical compounds: Rate constants and elimination in various water matrices. Chemosphere, 77(1), 53-59. https://doi.org/10.1016/j.chemosphere.2009.05.035

León-Ramírez, S. (2002). Shigelosis (disentería bacilar). ... Supervivencia En Recién Nacidos de Muy ..., 8(1405-2091), 5.

Leung, Y. L. (2014). Staphylococcus aureus. In Encyclopedia of Toxicology: Third Edition. https://doi.org/10.1016/B978-0-12-386454-3.00539-X

Mackenzie, E. F. W., Taylor, E. W., \& Gilbert, W. E. (2009). Recent Experiences in the Rapid Identification of Bacterium coli Type I. Journal of General Microbiology. https://doi.org/10.1099/00221287-2-2-197

Magdaleno, A., Juárez, Á. B., Paz, M., Tornello, C., Núñez, L., Moretton, \& Juan. (2012). Evaluación ecotóxica y genotóxica de aguas residuales hospitalarias. Acta Toxicol. Argent.

Marín, R., Ripollés, F., Santateresa, E., Lahora, A., González, I., Mantecón, R., \& Rodríguez, R. (2009). Contaminación convencional, sustancias prioritarias y contaminantes emergentes en saneamientos públicos españoles. Tecnología Del Agua.

Mosquito, S., Ruiz, J., Bauer, J. L., \& Ochoa, T. J. (2011). Mecanismos moleculares de resistencia antibiótica en Escherichia coli asociadas a diarrea. Revista Peruana de Medicina Experimental y Salud Pública. 
Pablo García Blancas1, A. M. M. (2014). Pruebas bioquímicas tradicionales y de alta resolución para identificación manual de enterobacterias. Acta bioquímica clínica latinoamericana.

Pupo, G. M., Lan, R., \& Reeves, P. R. (2002). Multiple independent origins of Shigella clones of Escherichia coli and convergent evolution of many of their characteristics. Proceedings of the National Academy of Sciences. https://doi.org/10.1073/pnas.180094797

Ramírez-Sánchez, I. M., Martínez-Austria, P., Quiroz-Alfaro, M. A., \& Bandala, E. R. (2015). Efectos de los estrógenos como contaminantes emergentes en la salud y el ambiente. Tecnologia y Ciencias Del Agua.

Ramos Alvariño, C. (2009). Medicamentos de consumo humano en el agua, propiedades físico-químicas. Revista Cubana de Higiene y Epidemiologia.

Tejada Llacsa, P. J., Huarcaya, J. M., Melgarejo, G. C., Gonzales, L. F., Cahuana, J., Pari, R. M., ... Chacaltana, J. (2015). Caracterización de infecciones por bacterias productoras de BLEE en un hospital de referencia nacional. Anales de La Facultad de Medicina. https://doi.org/10.15381/anales.v76i2.11143

Terrier, B., \& Martinez, V. (2012). Salmonelosis. EMC - Tratado de Medicina. https://doi.org/10.1016/s1636-5410(06)70402-0 


\section{PARA CITAR EL ARTÍCULO INDEXADO.}

Beltrán Dávalos, A., Escudero Vilema, M., Córdova Morales, S., \& Rosero Erazo, C. (2019). Monitoreo microbiológico para la gestión ambiental de Aguas Residuales Hospitalarias. Ciencia Digital, 3(3.4.), 242-253. https://doi.org/10.33262/cienciadigital.v3i3.4.882

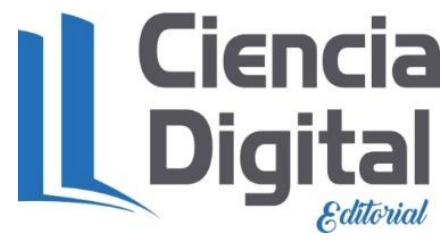

El artículo que se publica es de exclusiva responsabilidad de los autores y no necesariamente reflejan el pensamiento de la Revista Ciencia Digital.

El artículo queda en propiedad de la revista y, por tanto, su publicación parcial y/o total en otro medio tiene que ser autorizado por el director de la Revista Ciencia Digital.
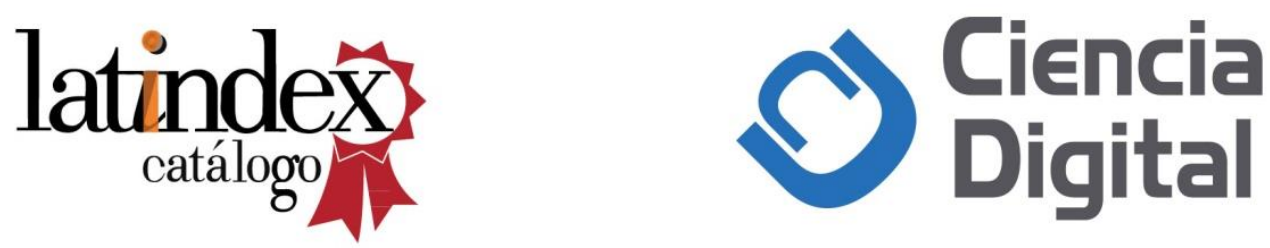